\title{
Fingertip force, surface geometry, and the perception of roughness by active touch
}

\author{
SUSAN J. LEDERMAN* and M. M. TAYLOR $†$ \\ Defence and Civil Institute of Environmental Medicine \\ Downsview, Ontario, Canada
}

Ss made magnitude estimates of the perceived roughness of grooved metal plates under conditions of active touch with controlled finger force. The wider the grooves in the plate, the narrower the lands between the grooves, or the greater the finger force, the greater was the perceived roughness. Increase in finger force also slightly increased the slope of the magnitude estimation function, suggesting not only that the roughness of a uniform surface but also the contrasts in the roughness of differing parts of a patterned surface would be altered by changes in the manner of feeling the surface. An analogous effect has been reported in vision, in that increases in illumination increase the apparent contrast of a surface.

The world we live in is primarily a visual one. We use our eyes to tell us about the shape of an object, its size, its color, and its orientation. The information is obtained rapidly and usually proves amazingly accurate. The visual modality plays such a dominant and dominating (e.g., Rock \& Harris, 1967) role in our world, however, that it is often easy to overlook the importance of tactile exploration. The touching process tells us a good deal about the more intimate qualities of things, such as temperature and surface texture-roughness, hardness, oiliness, stickiness, slipperiness, etc. A tailor examines the quality of a piece of cloth by passing it between his fingers; a housewife assesses the ripeness of a piece of fruit on the basis of its firmness or hardness, and one usually decides how to proceed across a slippery looking part of the sidewalk by stepping along it carefully at first and "feeling it out," to determine the degree of friction between shoe and ice.

In recent years, there has been an increasing interest in haptic (active touch) perception as opposed to perception by passive touch (e.g., Gibson, 1962, 1966). However, most research has dealt with the ability of persons to determine the spatial characteristics of objects. Vision is the modality most suited to such tasks, and, in general, the findings indicate that, although the visual and tactual percepts tend to yield basically the same results, touch is usually the less accurate of the two for spatial perception.

*This paper is based on a thesis by the senior author to be submitted to the University of Toronto in partial fulfillment of the requirements for the $P h D$ degree.

TThis is DCIEM Research Paper No. 824. For reprint requests, write to Defence and Civil Institute of Environmental Medicine, Civil Institute of Environmental Medicine,
P.O. Box 2000, Downsview, Ontario, Canada.
Less work has been done on aspects of perception unique to the touching system. Stevens and his associates (e.g., Stevens \& Harris, 1962; Stevens, J. C., \& Stevens, S. S., 1960) for example, have used magnitude scaling techniques on various tactile dimensions, including roughness. The usual linear relation between (log) physical stimulus and (log) perceptual response found in magnitude scaling studies has been found in these dimensions. However, the studies have generally been concerned with the magnitude estimation process rather than with the tactile percept itself.

In our view, "active" and "passive" touch do not constitute a dichotomy, as suggested by Gibson (1962). Rather, the touching process may be considered more or less "active," depending upon the degree of control that the $\mathrm{S}$ has over the various components of the touching process. To study the effect upon perceived roughness of one such component, fingertip force, we reduced the $S$ 's control over the force with which he pressed down on the surface he was examining. In the two experiments reported here, the effect of the geometrical characteristics of the surfaces upon the perception of roughness was also of primary interest.

This paper represents a first attempt to define stimulus characteristics relating to the percept of "roughness."

\section{EXPERIMENT 1 \\ Method}

Apparatus and stimuli. 1 The apparatus used to control finger force was designed along the lines of the classical balance scale (Fig. 1). As long as the balance is steady, the moments of the two arms must be equal, and, hence, the fingers must exert a well-defined force to balance the weight on the other arm of the balance. The constancy of the force of the fingers on the stimulus arm is disturbed only by small acceleration forces as the arm moves up and down. This variation is a small proportion of the total force exerted by the fingers. In simulation tests, we substituted a force gauge for the $S$ 's fingers and found that the "finger" force usually remained within about $20 \%$ of its nominal value.

The stimulus object was a smoothed aluminum alloy plate, $5 \frac{1 / 2}{2} 4^{1 / 2} \times$ $3 / 16$ in., scored with a set of parallel grooves, parallel to the line of the balance arm, and extending across the width of the central third of the plate. The plate fitted into a tray that could be fixed at any position along one arm of the balance, permitting $\mathbf{E}$ to adjust for differences in the arm length of Ss. A free-swinging pan hanging under the tray held removable weights that were used to alter the finger force required within any trial.

The stimuli were cut by a machine (shaper), which forms accurate linear grooves. The grooves were cut so as to leave $0.010 \mathrm{in}$. $(.25 \mathrm{~mm})^{2}$ of undisturbed surface (a "land") between each pair of grooves (Fig. 2). The groove depth was always $0.005 \mathrm{in}$. $(.125 \mathrm{~mm})$, and different plates were cut with different groove widths, varying from $0.005 \mathrm{in}$. $(.125 \mathrm{~mm})$ to 0.040 in. ( $1 \mathrm{~mm}$ ) in nominal increments of $0.005 \mathrm{in}$. $(.125 \mathrm{~mm})$. The shaper could cut a groove or land to within 0.002 in. $(.05 \mathrm{~mm})$ of the nominal value. The plates of the first set cut were numbered from 1 to 8 , where plate number $k$ had a nominal groove width of $0.005(.125) \times \mathrm{k}$ in. (mm). The "pitch" (land + groove) of the first set of plates thus varied from 0.015 in. (.375 mm) (Plate 1) to 0.050 in. (1.25 mm) (Plate 8). The groove and land widths were measured using a traveling microscope, and these "true" values are used in the figures to follow. After grooving, all plates were shaved on the reverse side to equate their weights. For obvious reasons, we would have preferred to use sinusoidally cut tiles but could find no technologically feasible way to produce them. Even the tiles we did use were difficult and expensive to produce. Stimulus control is a major barrier to the effective investigation of touch.

Experimental procedure. The $\mathbf{S}$ sat on a stool beside the apparatus, his right elbow resting comfortably in a shaped padded armrest mounted on a swivel over the fulcrum of the balance arm. The stimulus side of the balance was occluded from the S's view by a curtain. The stimulus tray was fixed in such a position that the tips of the S's bent fingers would rest on the middle of the stimulus plate when the balance 

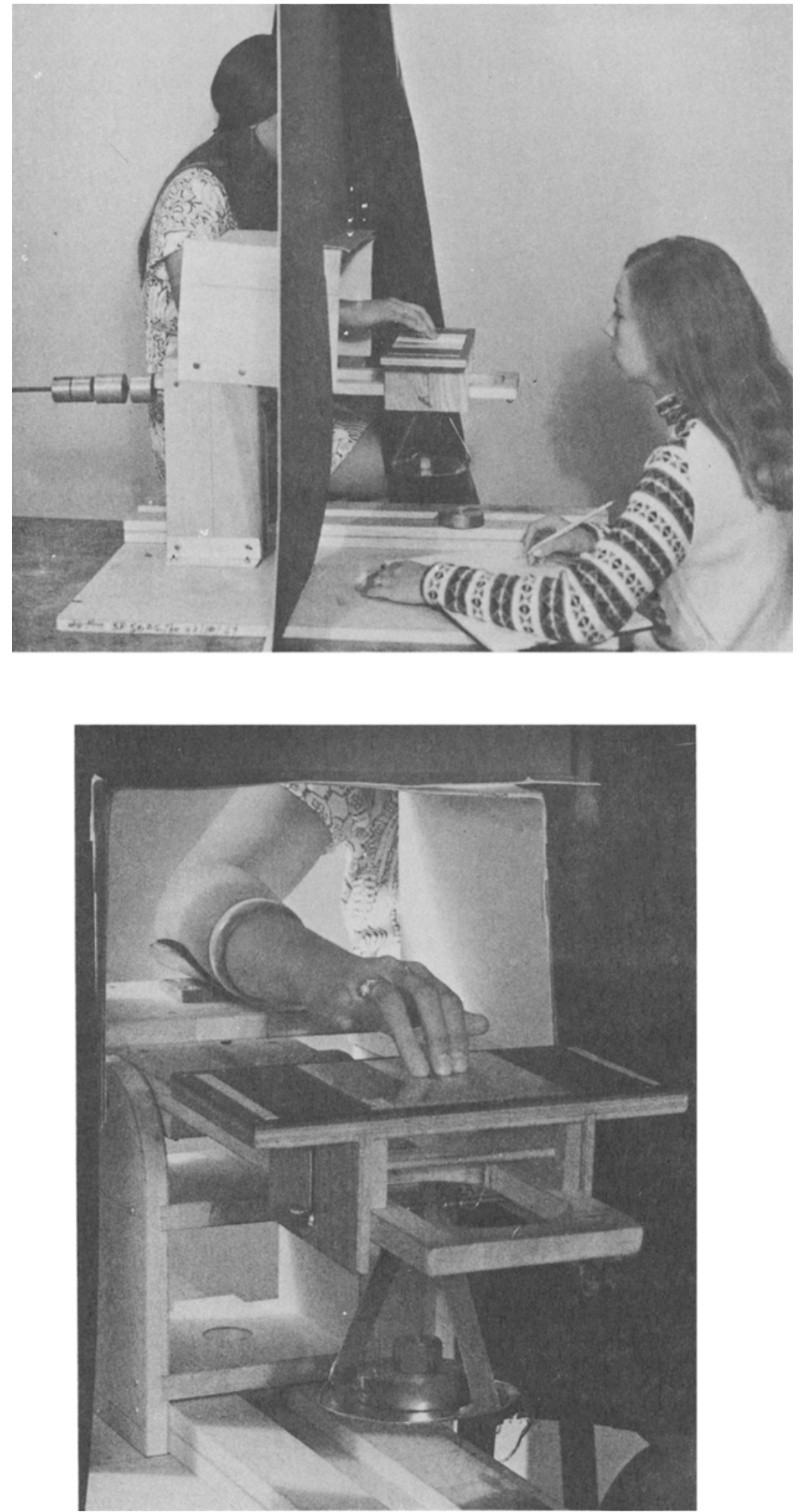

Fig. 1. (a) A side view of the apparatus. The $E$ is seated at the front end of the apparatus. The $S$, on the E's right, has placed her three middle fingers on the grooved portion of the tile to indicate when the balance arm is in a level and steady position. The $\mathbf{E}$ may adjust the force by adding to or removing weights from the pan suspended under the stimulus tray. (b) A front view of the same apparatus. arm was level. With all the weights in place in the pan under the tray, the counterweights at the other end of the balance arm were then adjusted to provide a net upward force of $1 \mathrm{oz}$ $(\cong 28 \mathrm{~g})$ at the middle of the plate. This was the total force the S's fingers had to apply in order to keep the balance arm steady and level. Weights could be removed from the pan to increase the finger force required to. keep the balance even. The finger forces used were $1(\cong 28 \mathrm{~g}), 5$ $(\cong 140 \mathrm{~g})$, and $25(\cong 700 \mathrm{~g}) \mathrm{oz}$. The balance apparatus was explained to the $S$, who was told that he must exert enough force to keep the balance arm steady and level at all times while he was feeling the roughness of the grooved surfaces. After he had thoroughly washed his hands and dried them in an airjet, he began the experiment.

The experiment was one of magnitude estimation. The $S$ was told that he must give a number corresponding to the roughness of each of the stimuli and that he should ignore the changes in force when making his judgments of roughness. Neither standard nor prespecified modulus was used for the magnitude estimation. Instead, before the run started, two practice stimuli were given to generally define the range of roughness that the $S$ should expect and to permit him to establish for himself a range of response numbers that could include any positive (nonzero) value. Examples of possible range values, such as 1 to 10 , or 10 to 100 , were suggested to each $S$. In practice, the highest range used was 60-950 and the lowest was 0.24-5.0. It was pointed out to the $S$ that there was an infinite set of possible numbers that he might use between 0 and 1 . The two practice stimuli were Plate 1 with low finger force (described as "one of the roughest..."). Ss were told that they might well be presented with tiles that felt rougher or smoother than the two practice plates. They should feel free, therefore, to use numbers above or beyond the range of responses chosen, when such an event as this occurred.

When feeling the stimuli, the $\mathbf{S}$ was instructed to use only the tips of his three middle fingers, not the pads or nails. No motions along the grooves in the plate were allowed. The $S$ could move his fingers back and forth (in the horizontal plane) across the stimulus grooves at any rate he wished, but he was not permitted to hold the stimulus down with one or two stationary fingers while exploring it with the other(s). If he were to do so, he might feel the grooves at a force greatly different from that intended, the stationary fingers taking the weight off the active one. 
Whenever he wished, the $\mathbf{S}$ made his estimate and released the balance. $E$ then changed the stimulus plate, added or subtracted weights on the pan, and the next trial began. All runs consisted of 24 trials. Each of the eight plates was used at each of the three finger-force settings $[1(\cong 28 \mathrm{~g}), 5$ $(\cong 140 \mathrm{~g})$, and $25(\cong 700 \mathrm{~g}) \mathrm{oz}]$. The $S$ was permitted a short rest at any time if he felt that his fingers were becoming less sensitive or unduly sweaty.

After the first run, there was a 10-min break, during which the $S$ again washed and dried his hands and after which the second run was begun. Between runs, the $S$ was permitted to change the range of response numbers that he wished to use. He was not allowed to change the range within runs. One complete session of two runs lasted approximately $40 \mathrm{~min}$. After each session, the tiles were cleaned with trichloroethylene solvent, using a typewriter brush to remove any embedded particles.

Experimental design. The experiment was treated as a factorial design, the factors being the 8 Ss, 8 plates, 3 finger forces, 2 runs within each experimental session, and 2 separate sessions (i.e., days) for each $\mathbf{S}$. The presentation order of the 24 conditions (Plates by Forces) during each run was determined by $E$, who filled in the 8 by 3 stimulus matrix the single stipulation that Plates 1 and 8 never immediately follow each other.

Subjects. There were four male and four female Ss, all right-hancied and all employed in various capacities at this (Tiles by Forces) haphazardly, with

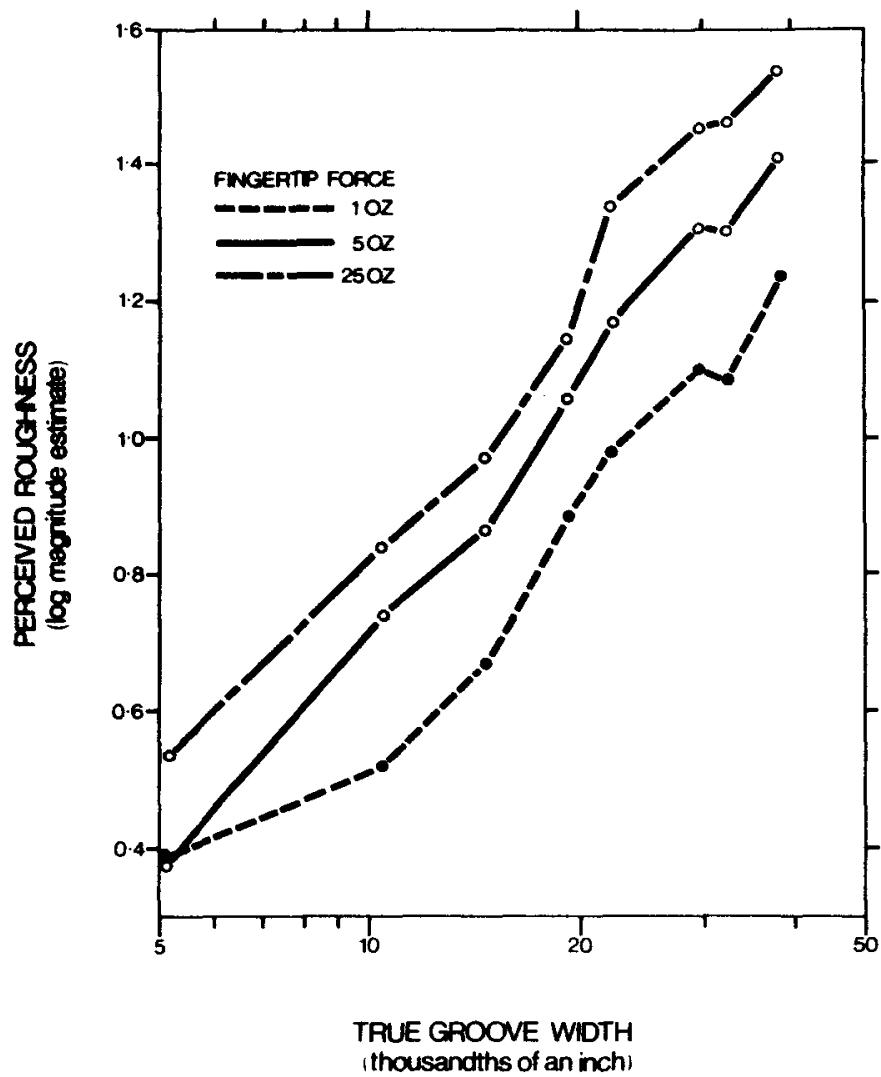

Fig. 3. Experiment 1: Magnitude estimate (roughness) as a function of "true" groove width and fingertip force. institute. Their ages ranged from 19 to 50 years. None knew the purpose of the experiment when initially tested, although most had previously served as Ss in other psychophysical experiments.

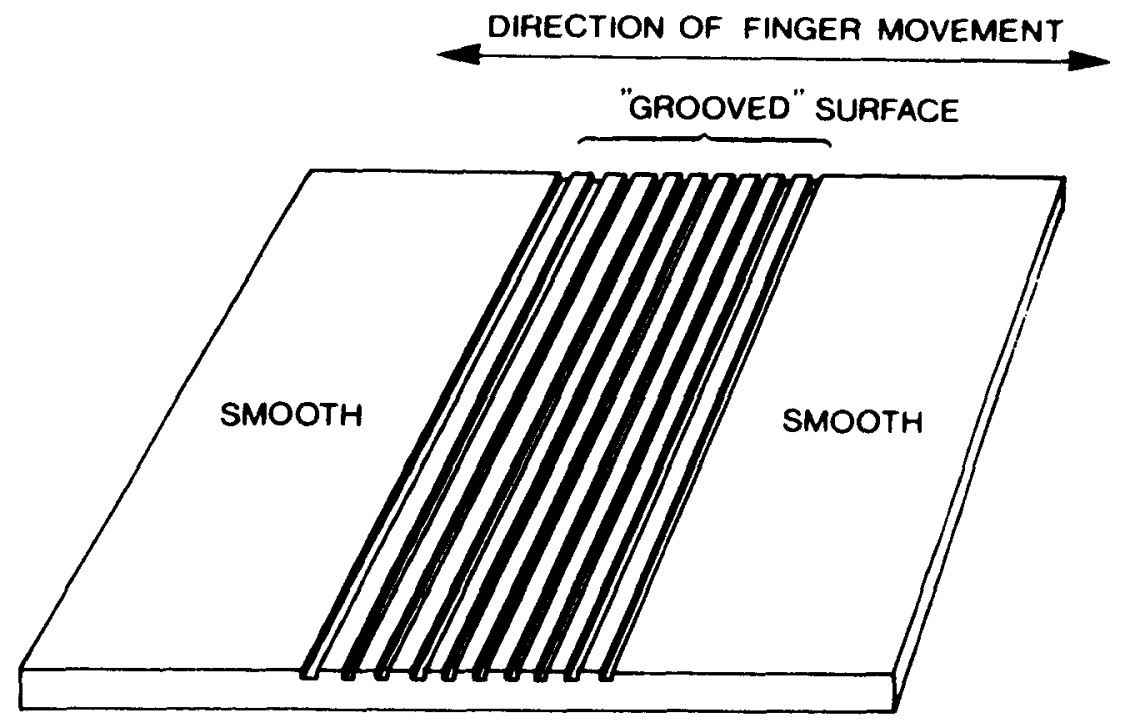

Fig. 2. A grooved aluminum plate. The outer thirds of the plate are smooth; the inner section has parallel linear grooves cut along the width of the plate.
An analysis of variance was performed on the data, using a completely crossed five-factor design, the factors being $S s$, days, runs, groove widths, and forces. The most striking result is that fingertip force and groove width affect the perceived roughness of the grooved surfaces $(p<.001)$. Figure 3 shows the relation of roughness to groove width and fingertip force. As all the data were logarithmically transformed before analysis, only the log (magnitude estimates) are reported here. Log (magnitude estimate) of roughness is plotted as a function of log ("true" groove width) for each of the three forces. The plotted groove width values are the measured rather than the nominal sizes. Each data point represents the average of 32 responses ( $8 \mathrm{Ss}, 2$ days, 2 runs/day). As can be seen in Fig. 3 , the greater the force, the rougher the tiles feel. The exact form of the relation between groove width and apparent roughness, however, is unclear. A straight line, which would represent the conventional (e.g., Stevens, 1957) power law, is an adequate representation of most of the data for each force.

The analysis of variance showed a 


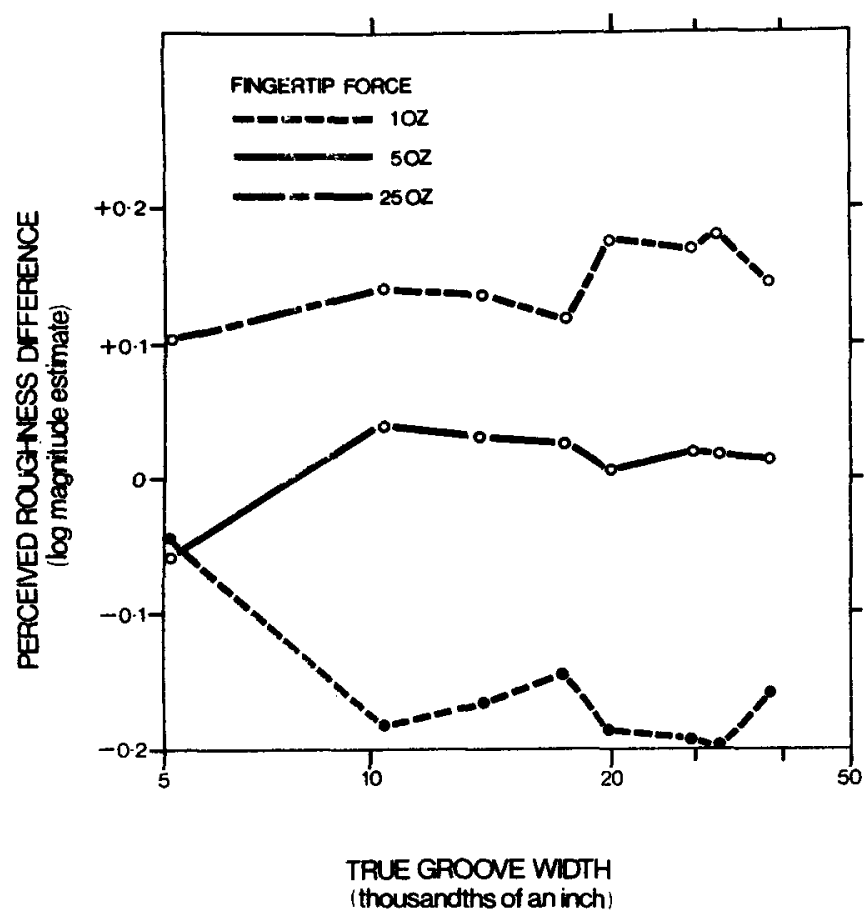

Fig. 4. Experiment 1 : Difference in magnitude estimate (roughness) between (1) mean roughness of groove widths averaged over fingertip force conditions, and (2) mean roughness of corresponding groove widths presented at each force level as a function of "true" groove width and fingertip force.

Groove Width by Finger Force interaction term that was highly significant $(p<.001)$. The interaction proved due to a slight divergence of the curves for roughness vs groove width, which may be better seen in Fig. 4. The difference between (1) the mean scores for each groove width averaged over all forces and (2) the individual score for that same groove width at each force level is plotted as a function of groove width. It is apparent that, as groove width increases, there is an increasing contrast in the roughness of a grooved plate when using the three different forces. The $S$ by Groove Width interaction, which is of scant theoretical interest, also proved significant ( $p<.001)$, though small. Of course, main effects, other than those already plotted for groove width and pitch, are of no interest whatsoever, because the Ss changed their response ranges from session to session.

Beyond the simple determination of perceived roughness, we were interested in determining whether the precision of the Ss' responses was influenced by the particular groove width and/or finger force.

A computer program was written ${ }^{3}$ to perform what we have called ANOVAVA, or analysis of variance of variance (described in the Appendix). ANOVAVA analyzes the patterns of precision in the data. The results indicated that, as one might expect, there were differences in the precision with which various Ss responded. These differences were not correlated with sex, nor did groove width, force, or the interaction of the two have significant differential effects upon the precision with which the Ss judged the roughness of the plates. 'It was unfortunately impossible to assess accurately the correlation between precision and the range of numerical responses chosen, since Ss could change their scales both within and across days.

\section{EXPERIMENT 2}

In the first experiment, it was impossible to separate out the effects of groove width on perceived roughness from those due to pitch. Within this set of tiles, an increase in groove width implies an equal increase in pitch, since all the lands are equal. To eliminate this ambiguity, a second experiment was run in which two sets of tiles (eight in each set) were used: pitch remained constant between corresponding pairs of tiles; groove width increased (lands constant) in one set ("groove set") and land width varied similarly (grooves constant) in the other ("land set"). Each tile was thus a member of a pair having the same pitch.

The groove set was a modified version of the set used in Experiment 1, consisting of the original Plates 1-5 from Experiment 1 plus three new plates cut to duplicate the groove widths of Plates 6-8, but with a new groove depth of 0.010 in. $(0.25 \mathrm{~mm})$. The modification was made because we thought that the apparent depression of the roughness of Tiles 6,7 , and 8 might be due to the finger touching the bottom of the wider grooves. We name the groove-set tiles of this experiment G1 to G8. For the land set, the groove depth and width were held constant at 0.005 in. $(0.125 \mathrm{~mm})$ and $0.010 \mathrm{in} .(0.25 \mathrm{~mm})$, respectively; the lands varied from 0.005 in. $(0.125 \mathrm{~mm})$ to $0.040 \mathrm{in}$. $(1.0 \mathrm{~mm})$, in 0.005 -in. $(0.125-\mathrm{mm})$ nominal increments, giving a pitch series the same as that for the groove set. These tiles are known as L1 to L8. Tile L2 was actually the same tile as G2. G1 was paired with L1, G3 with L3, G4 with L4, and so forth. The members of each pair had the same pitch.

If pitch were the most important variable affecting apparent roughness of the tile surface, then the roughness should be similar for the two members of each pair; if groove width were the primary variable, then the roughness of all the tiles in the land set should be roughly the same, whereas that for the tiles of the groove set should vary, as was shown by Experiment 1. Finally, if land width were another effective variable, then differences among the land set should be evident, but the pairs need not match. Note that the pitch determines the frequency of the vibrations set up in the skin by the tile.

\section{Method}

Subjects. There were five male and four female Ss, all right-handed and all employed in various capacities at this institute. Their ages ranged from 20 to 34 years. One of the Ss had participated in the first experiment. The others did not know the purpose of the experiment, although most of them had had previous experience in psychophysical experiments.

Procedure. We used the same procedure as that of Experiment 1 but with minor modifications. (1) Ss now dried their hands in the warm air provided by a commercial portable hair drier. (2) At the beginning of each trial, $E$ steadied the balance arm and placed the S's fingers on the smooth portion of the left side of the tile. $E$ released the balance arm, the $\mathbf{S}$ balanced it himself, and, when ready, began to feel the grooved stimulus surface. In Experiment 1, the $S$ had placed his fingers on the grooved part of the surface and then pushed down on it to balance the arm properly 


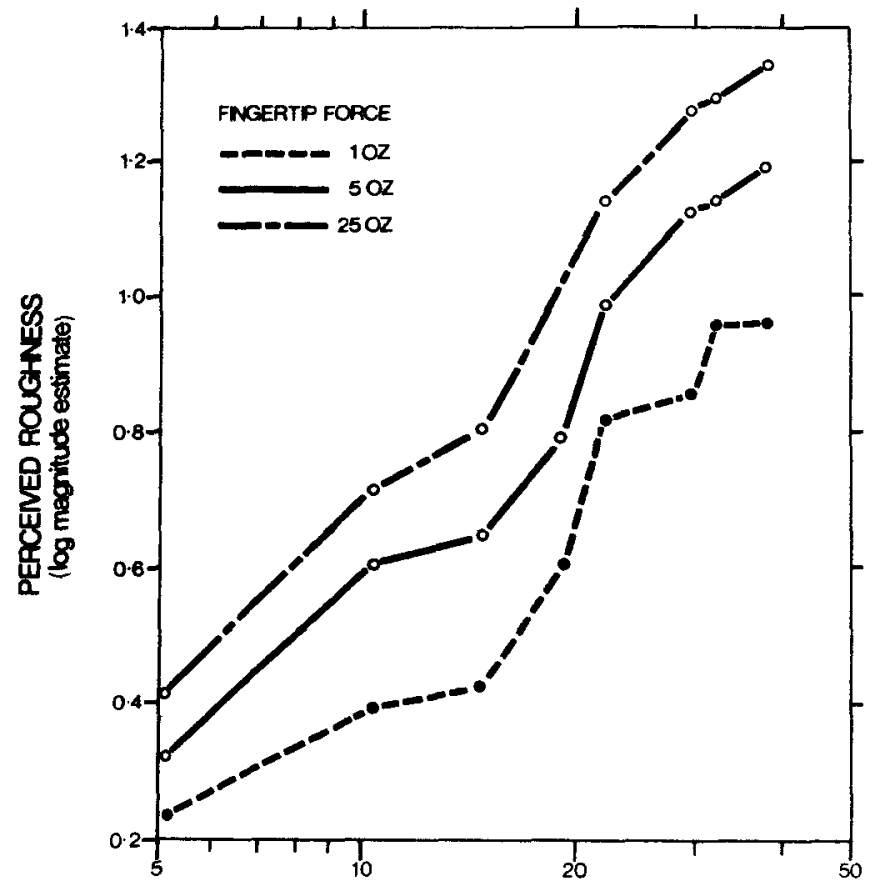

TRUE GROOVE WIDTH (thousandths of an inch)

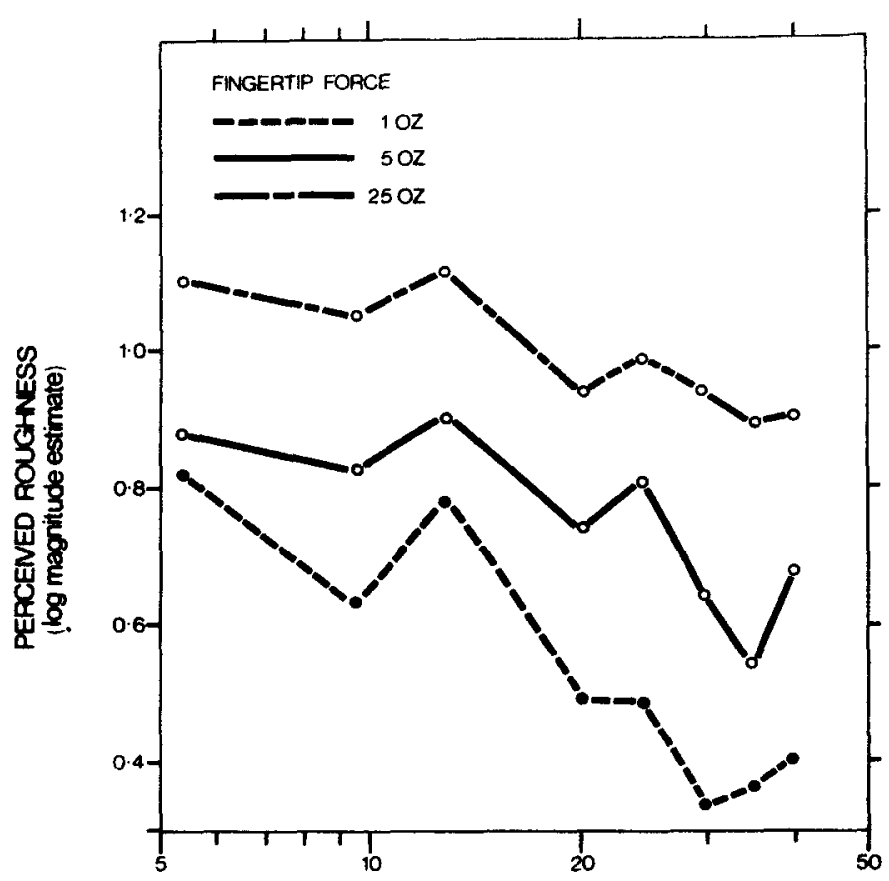

TRUE LANO WOTH

(thousandths of an inch)

Fig. 5. (a) Experiment 2: Magnitude estimate (roughness) as a function of "true" groove width and fingertip force. (b) Experiment 2 : Magnitude estimate (roughness) as a function of "true" land width and fingertip force.

before beginning to move his fingers across the tile. But in Experiment 2, the $S$ obtained information as to the roughness of the grooved portion only while his fingers were moving. (3) The introductory tiles that were used to indicate the range for the land ret were difficult to choose because it was obvious from preliminary observations that the relation between apparent roughness and land width was not an increasing monotonic function, as it is for groove width. Not knowing which stimuli of the land set would prove roughest or smoothest, we chose to present both L1 and L8 at both $1 \mathrm{oz}$ and $25 \mathrm{oz}$. They were described as "four examples of the tiles you will feel."

Experimental design. Ss were run a total of 4 days (not necessarily consecutively). Either the land set or the groove set was presented during the first 2 days, followed by the other set on the final 2 days. The two sets of stimuli were not mixed together for two reasons. First, even if we wanted to mix the sets, it would have been impossible to run 96 trials on the same day, because of finger "fatigue." Second, we felt that to perform a proper replication of the first experiment for variable groove widths, we had to avoid the possible biasing effects that the small roughness differences between the tiles of the land set might have had on the range of numbers that would otherwise be applied to the more widely varying tiles of the groove set. The effects of such biasing in task related to magnitude estimation has been discussed by Stevens (1957) and demonstrated for loudness by Pollack (1964).

A completely crossed factorial design was used, and the data for the groove set and land set were analyzed separately, each as in Experiment 1. The factors analyzed were: Ss (9), groove width (or land width) (8), days (2), runs (2), and force (3).

\section{Results}

In Figs. 5a and 5b, log (magnitude estimate of roughness) is plotted as a function of $\log$ ("true" groove width) and log ("true" land width), respectively, for each of the three force conditions. The three ascending curves in Fig. 5a represent the results of varying groove width for the three forces: the descending curves in Fig. 5b represent the effect of land width and force on perceived roughness.

An analysis of variance performed on the groove-set data indicates that, as in Experiment 1, apparent roughness was strongly affected by fingertip force $(p<.001)$ (Fig. 5a). The three force functions were again not parallel, as indicated by the highly significant Groove Width by Force interaction $(p<.001)$. The analysis of variance (not shown in this report) performed on the data from the land set indicated strong effects due to land width and force $(p<.001)$ (Fig. 5b). The significant nonparallelism of the force functions for the groove-set and land-set data is shown in Figs. $6 a$ and $6 \mathrm{~b}$, respectively.

No direct comparison may be made between the "land" and "groove" functions regarding differences between absolute roughness scores because Ss were allowed to change their number systems across days and runs, but the slopes may be compared.

Inspection of the two sets of curves in Figs. 5a and 5b indicates that pitch is not an important factor in the determination of the roughness percept for these tiles. If it had been, the two sets should have the same slopes. However, perceived roughness actually decreased with increasing land widths, the size of decrease being dependent upon the force used $(\mathrm{p}<.001)$. The positive slopes are much steeper for the groove set than are the negative slopes for the land set, suggesting that although both variables influence apparent roughness, groove 

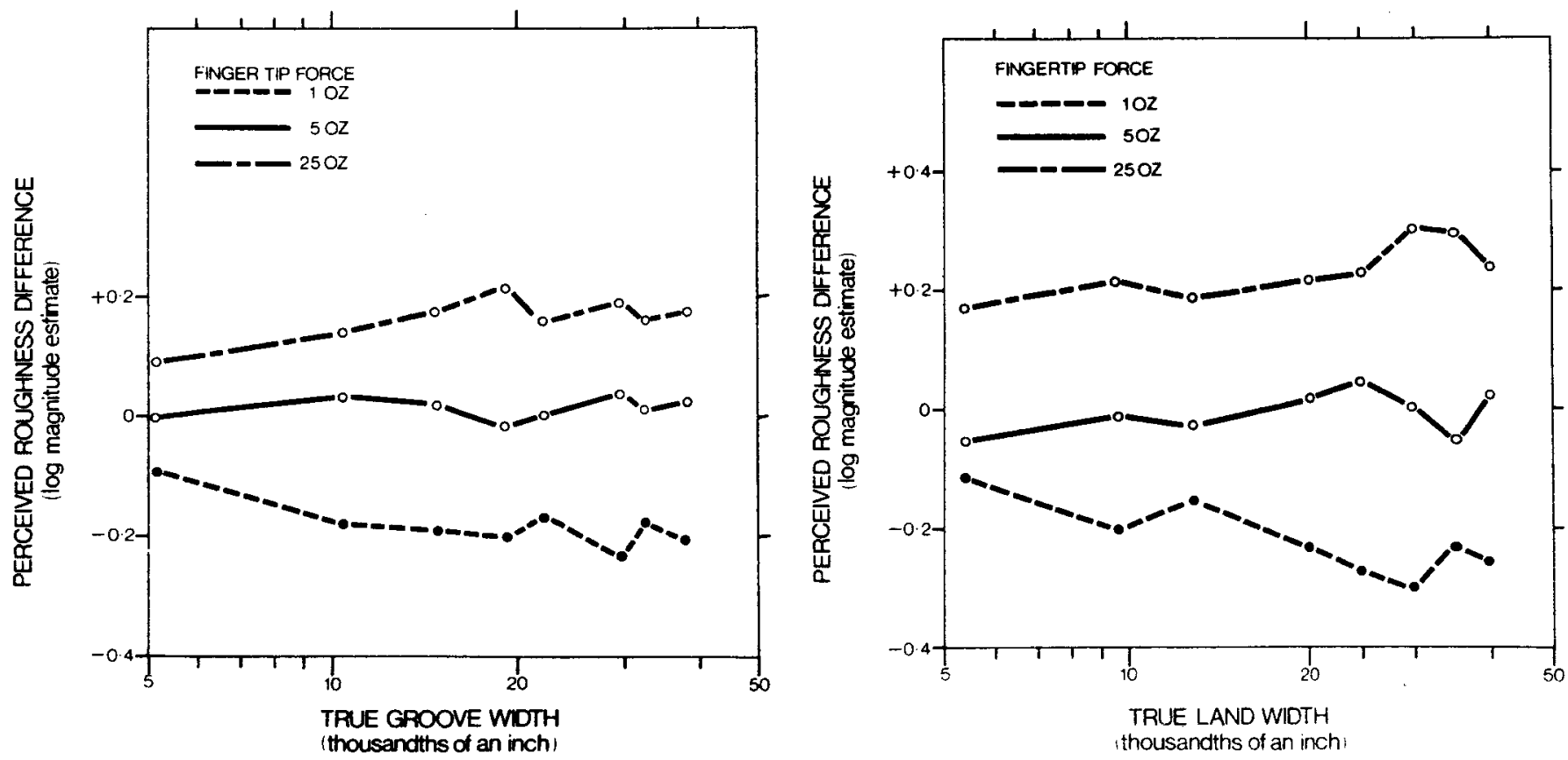

Fig. 6. (a) Experiment 2: Difference in magnitude estimate (roughness) between (1) mean roughness of groove widths averaged over fingertip force conditions, and (2) mean roughness of corresponding groove widths presented at each force level as a function of "true" groove width and fingertip force. (b) Experiment 2: Difference in magnitude estimate (roughness) between (1) mean roughness of land widths averaged over fingertip force conditions, and (2) mean roughness of corresponding land widths presented at each force level as a function of "true" land width and fingertip force.

width is the more influential of the two. On the other hand, it may be seen (Figs. 3, 4, 5a, and $5 \mathrm{~b}$ ) that the roughness contrast of pairs of tiles at different forces does seem to increase somewhat with increasing pitch, i.e., the relative differences in magnitude estimates of roughness are larger for the wider lands or grooves than for the narrower ones. Irregularities in the curves, particularly for the land set, are possibly due to idiosyncracies in machining of the individual tiles.

It would seem reasonable, on the face of it, to combine the absolute levels of the results for the land set with those for the groove set, through the common tile known as L2 or G2. This cannot be done, however, because of the "stimulus range effect" (Poulton, 1968), which expands the differences in magnitude estimates when the stimulus range is small. This effect can be seen in the effect of finger force on the roughness for L2, which is greater than the same effect for G2. It was in anticipation of this effect that we did not conduct the land experiment mixed with the groove experiment. The excessive number of smooth tiles we would have introduced would have been expected to enhance the slope of roughness vs groove width (e.g., Stevens, 1957; Pollack, 1964).

\section{DISCUSSION}

The two experiments reported here demonstrate that there is an effect of fingertip force upon the perceived roughness of a grooved surface. As the force increases, so too does apparent roughness. At first sight, this finding might seem to be directly related to finding that the perceived magnitude of a vibration increases as the force of the contactor on the skin increases (Craig \& Sherrick, 1969). However, Craig and Sherrick used a fairly large contactor area ( $4 \mathrm{~mm}$ in diam), and other studies (e.g., Verrillo, 1963, 1971) have shown other integrative effects of vibratory amplitude to disappear with small contactor areas (e.g., $1 / 2 \mathrm{~mm}$ up for the frequencies of interest to us). The effects of roughness in this study must depend on discriminations within very small areas which are always less than Verrillo's minimum integrating areas. Hence, any application of Craig and Sherrick's result to the effect of force on the roughness of our tiles is rather dubious.

We have chosen extreme force values to determine the limits of the force effect. A person using three fingers can apply no more than approximately $25 \mathrm{oz}$ of force while still being able to move smoothly across the tiles used in these experiments. With light forces, our apparatus cannot effectively set the fingertip force at less than $1 \mathrm{oz}$, since this nominal value is altered by the acceleration forces of the balance arm as it moves up and down. Furthermore, with the present technique, it was physically possible for a person to support the force of the balance arm against the outer two fingers while using the middle fingertip to explore the surface texture with less force than $E$ intended. Such tactics were prevented to some extent by instructions and by the vigilance of $E$.

In addition to force, several characteristics of the surface grooves in the aluminum tiles affected the perception of roughness; the width of the groove and the land influenced perceived roughness, but to different degrees and in different directions. Increasing groove width resulted in increasing roughness, whereas increasing land width resulted in decreasing roughness, although the differences were a great deal less than those brought about by varying groove width. The inverse effects permitted us to rule out pitch as the single most influential factor on the perceived roughness of the grooved plates. The inverse relation between the effects of groove width and of land width suggests the possibility that groove to pitch ratio might be a key variable affecting perceived roughness. However, when roughness is plotted as a function of this ratio (not shown here), it proves to be a rather poor predictor.

Pitch seems to have a different but interesting effect of its own. As pitch 
increases (Figs. 3, 5a, and 5b), so too does the difference between the apparent roughnesses of a surface at two forces. This is apparent in all three sets of curves, with land or groove width increasing.

Stated another way, perceived roughness as a function of groove width increases faster with high finger force than it does with low force. This change in slope is real, as is shown by the numerical values given in Table 1 . This implies that the roughness contrast of a patterned surface should increase with increasing finger force. There may be an analogous effect in vision. Light surfaces increase in brightness faster with increased illumination than do dark surfaces (Jameson \& Hurvich, 1961; Flock \& Noguchi, 1970), so that the apparent contrast of a scene increases with illumination.

Finally, one must consider that the maximum forces used in these experiments are unrealistic. It is most unlikely that a person would freely use $25 \mathrm{oz}(\sim 700 \mathrm{~g})$ of force to examine the roughness of an object. A logical extension to the experiment would be to permit the $S$ total control over the fingertip force and also, therefore, over the entire touching process. We would like to know what force the $S$ does use, whether he alters it during his tactual explorations, and whether such alterations in force affect the roughness percept.

In respect to the initial aim of the study, which was to shed some light on the stimulus characteristics leading to the perception of "roughness," we can say with some certainty that "roughness" is dependent on the force applied between the fingertip and the surface and that it is not closely related to the frequency of vibratory impulses applied to the fingertip. Roughness seems to depend more on the distribution of regions over which the skin is and is not supported by the underlying surface than on the distribution of transitions between such regions. This is shown by the great difference in roughness between tiles with groove width and land width interchanged. A wide groove leads to a large roughness, a wide land to a small roughness. Beyond this, the results of the experiments reported here do not permit us to go.

\section{REFERENCES}

CRAIG, J. C. \& SHERRICK, C, E, The role of skin coupling in the determination of vibrotactile spatial summation. Perception \& Psychophysics, 1969, 6. 97-101.

FLOCK, H. R., \& NOGUCHI, $K$. An experimental test of Jameson and Hurvich's theory of brightness contrast. Perception \& Psychophysics, 1970, 8, 129-136.

GIBSON, J. J. Observations on active touch. Psychological Review, 1962, 69, 477-491.

Table 1

Slope of Log (Magnitude Estimate) as a Function of Log (Groove Width) or Log (Land Width)

\begin{tabular}{rcrc} 
& Experiment 1 & \multicolumn{2}{c}{ Experiment 2 } \\
\cline { 2 - 4 } & Grooves & Grooves & Lands \\
\hline $1 \mathrm{oz}$ & $1.01 \pm .08 *$ & $.92 \pm .11$ & $-.55 \pm .10$ \\
$5 \mathrm{oz}$ & $1.20 \pm .04$ & $1.05 \pm .08$ & $-.33 \pm .10$ \\
$25 \mathrm{oz}$ & $1.21 \pm .07$ & $1.12 \pm .06$ & $-.26 \pm .06$ \\
\hline
\end{tabular}

*One $S D$ limits listed. In each case the difference between the slope for 1 oz and that for $25 \mathrm{oz}$ is significant beyond the $5 \%$ level.

GIBSON, J. J. The senses considered as perceptual systems. Boston: Houghton Mifflin Company, 1966.

JAMESON, D., \& HURVICH, L. M. Complexities of perceived brightness. Science, 1961, 133, 174-179.

POLLACK, I. Neutralization of stimulus bias in auditory rating scales. Journal of the Acoustical Society of America, 1964, $36,1272-1276$.

POULTON, E. C. The new psychophysics: Six models for magnitude estimation. Psychological Bulletin, 1968, 69, 1-19.

ROCK, I., \& HARRIS, C. S. Vision and touch. Scientific American, 1967, 216, 96-104.

STEVENS, J. C., \& STEVENS, S. S. Warmth and cold: Dynamics of sensory intensity. Journal of Experimental intensity, Journal of Experis

STEVENS, S. S. On the psychophysical law. Psychological R eview, 1957, 64, 153-181.

STEVENS, S. S., \& HARRIS, J. R. The scaling of roughness and smoothness. Journal of Experimental Psychology, 1962, 64, 489-494.

VERRILLO, R. T. Effect of contactor area on the vibrotactile threshold. Journal of the Acoustical Society of A merica, 1963, 35, 1962-1966.

VERRILLO, R. T. Vibrotactile thresholds measured at the finger. Perception \& Psychophysics, 1971, 9, 329-330.

\section{NOTES}

1. We are most grateful to Mr. J. Royce and the members of his workshop for the time and energy spent in producing the apparatus and aluminum plates.

2. Since our machine shop works in the English system of measurement, all dimensions were produced in units of .005 in. nominal. Metric equivalents are reported in parentheses. 3. We thank Dr. D. M. Sweeney for
programming the ANOVAVA procedure.

(Received for publication January 6, 1972; revision received June $30,1972$.

\section{APPENDIX}

\section{ANOVAVA:}

The Analysis of Precision

An analysis of variance (ANOVA) permits one to assess the influence that each of several independent variables singly and conjointly have on the value of some dependent variable. The analysis of variance of variance (ANOVAVA) similarly allows one to assess the effects that the independent variables have on the precision with which the dependent variable can be determined. Just as the ANOVA takes as its data the raw measurements of the dependent variable, so the ANOVAVA uses for data the patterns of variation in the measurements. To perform an ANOVAVA, one attempts to assess the variance inherent in a measurement under a particular experimental condition and then performs an analysis of variance on the logarithms of these estimates. Hence, the name "analysis of variance of variance."

ANOVAVA has two separate uses. Firstly, it permits a test of the manner in which the data for an ANOVA depart from homoscedasticity and may suggest ways to separate the original data into separate, more sensitive, and justified analyses. Secondly, and more importantly, it permits one to assess what effect the experimental variables have on the precision of the dependent variable, which may be a theoretically important question. We recommend that ANOVAVA be carried out as a matter of course in conjunction with any ANOVA that contains a variable that promises to allow a sensible assessment of "within-cell" variance for the individual cells at some level of the ANOVA pattern.

The conceptual foundation of ANOVAVA is very simple, although there are some technical problems in its implementation. Briefly, the procedure is that one chooses an appropriate ANOVAVA design, say a factorial such as the $S$ by Groove Width (G) by Finger Force (F) design basic to the present study. One then adds a variable or variables intended to serve as replications; in the present study, we have Days (D) by Runs (R). These latter permit the assessment of the variation in the dependent variable (perceived roughness) under each unique combination of conditions of the experimental variables. There are problems in determining these individual variances, which we shall consider later. Once they have been determined, they are logarithmically transformed to make their distributions more nearly normal, and these log variances form the data for an ordinary ANOVA in the experimental variables ( $S$ by $G$ by F). ANOVAVA, then, consists of finding a factorial pattern of $\log$ variances and subjecting it to analysis as if the log variances had been raw data.

Two problems arise in implementing ANOVAVA. Both have to do with actually finding the most appropriate value for the log variance in a single cell. The first is a conceptual problem, relating to the fact that the variables intended to serve merely as replications may themselves affect the dependent variable. There may, for example, be leaming and fatigue effects. If the simple repeated scores were used to estimate the variance within a cell, these extraneous effects would be incorporated in the variance estimate, which would be inflated. Consistent effects such as these should be removed so far as possible from the variance estimates that go into the final stage of the ANOVAVA. One simple way to remove some extraneous effects is to ensure that the "replications" variables have more than one degree of freedom within each cell and that identifiable extraneous effects can 
be separated from the measurement error we are trying to determine. This can be done by having more than two replicates and removing a linear trend, or, as in the present case, by arranging the replicates so that they themselves form a factorial design (D by $R$ ) from which the interaction mean square may be extracted as an estimate of measurement error variance. Of course, the $D$ by $R$ interaction within any cell may itself contain real effects, but these can be eliminated only by comparisons among cells, a technique that introduces the possibility of spurious mutual effects within the final analysis.

The second major problem in determining the log variances for the final analysis derives from the quantized nature of the data. All data are quantized. In the present experiment, the quantization arises from the tendency of the $S$ to say " 20 ," whether he experiences $19.8774 \ldots$ or $20.3126 \ldots$.
Any single response must be considered to be an index to a distribution of underlying perceived magnitude. Under these circumstances, it is quite conceivable that the measured $D$ by $R$ interaction in some cell will be exactly zero, although the interaction due to the "true" perceived magnitudes would have been finite. Since the $\log$ of zero is minus infinity, zeros cannot be admitted as variance estimates. However, since a zero indicates only that the "true" interaction term is smaller than some ill-specified limit dependent on the somewhat variable quantization step size, it makes sense to replace the zero with some less biased estimate of that "true" interaction. We have chosen to use one-half the smallest nonzero variance estimate to replace all zeros before transforming logarithmically.

The full ANOVAVA procedure is then as follows: (1) construct an experimental design having experimental and replication vaxiables, where preferably the replication variables form a factorial pattern within each unique combination of the experimental variables (i.e., within each cell). (2) Make the best possible estimate of the measurement variance within each cell, preferably using the interaction term of a miniature ANOVA on the data points within the cell. (3) Logarithmically transform the variances thus determined, replacing all zeros with one-half the smallest nonzero variance before transformation. (4) Ferform a regular ANOVA over the space of the experimental variables, using the logarithmically transformed variances as data.

An ANOVAVA program has been written in FORTRAN for the PDP-9, and may be made available on request. 